\title{
Data Processing Techniques for Aberration-Corrected STEM
}

\author{
M.C. Sarahan, ${ }^{* * *}$ Q.M.Ramasse, ${ }^{* *}$ N.D. Browning, ${ }^{* * * * * * * * * *}$ \\ * Chemical Engineering and Materials Science, University of California, Davis, One Shields \\ Ave., Davis, CA 95616, USA \\ $* *$ \\ SuperSTEM Laboratory, STFC Daresbury, Keckwick Lane, Warrington, WA4 4AD, UK \\ *** Molecular and Cellular Biology, University of California, Davis, One Shields Ave., Davis, \\ CA 95616, USA \\ **** Condensed Matter and Materials Science Division, Physical and Life Sciences Directorate, \\ Lawrence Livermore National Laboratory, 7000 East Ave., Livermore, CA 94550, USA
}

With the advent of aberration-corrected scanning transmission electron microscopy, our ability to resolve details has begun to reach the limit imposed by quantum mechanics. This capability has enabled insight into the structure-function relationship behind superconductivity, catalytic activity and countless other critical material properties [1]. However, this increased resolution requires more careful image interpretation. In particular, the great increase in convergence angles reduces the depth of focus and can result in misleading images. With only part of the sample in focus, it becomes possible to explore depth-sectioning, but it also breaks the linear relationship between the atomic potential of an atomic column and its corresponding brightness in an image [2]. Image brightness is now tightly bound to defocus. The present state of aberration-corrected STEM is an intermediate between the projection imaging mode and confocal scanning microscopy, in which the assumptions of neither are wholly satisfied and image brightness must be interpreted with care.

In addition to complications arising from depth of focus, interpretation of the chemically sensitive Zcontrast images from high-angle annular dark field (HAADF)-STEM are hindered by thickness effects and noise (Figure 1). Though aberration correctors have vastly improved the signal-to-noise ratio in these images, this remains sufficiently problematic that most examples of identifying singleatom dopants have involved very large atomic number differences between the matrix and the dopant [2]. Multivariate image analysis provides objective, statistically sound, robust means of locating and quantifying small contrast differences [3]. Rather than examining columns individually, the technique compares unit cells throughout an image and derives characteristic brightness variations that occur throughout the image (Figure 2). By examining hundreds of unit cells, the confusing effects of noise can be excluded from analysis with greater confidence. Image registration using advanced computer vision algorithms allows this technique to be applied to multiple images from different sample regions or defocus settings simultaneously (Figure 3). This both improves the statistical reliability of the technique and introduces it as a useful means of threedimensional sample analysis.

\section{References}

[1] A.L. Bleloch et al., Proc. 14th Eur. Micr. Congress (2008) 1.

[2] A.R. Lupini et al., Microsc. Microanal. 15 (2009) 441.

[3] P. Trebbia and N. Bonnet, Ultramic. 34 (1990) 165.

[4] Work supported by the Materials Design Institute, Los Alamos National Laboratory, LANS Contract 25110-002-06, Mod 6; and University of California Lab Management Fees Award. The aid of Dr. David Morgan of Indiana University is gratefully acknowledged. 


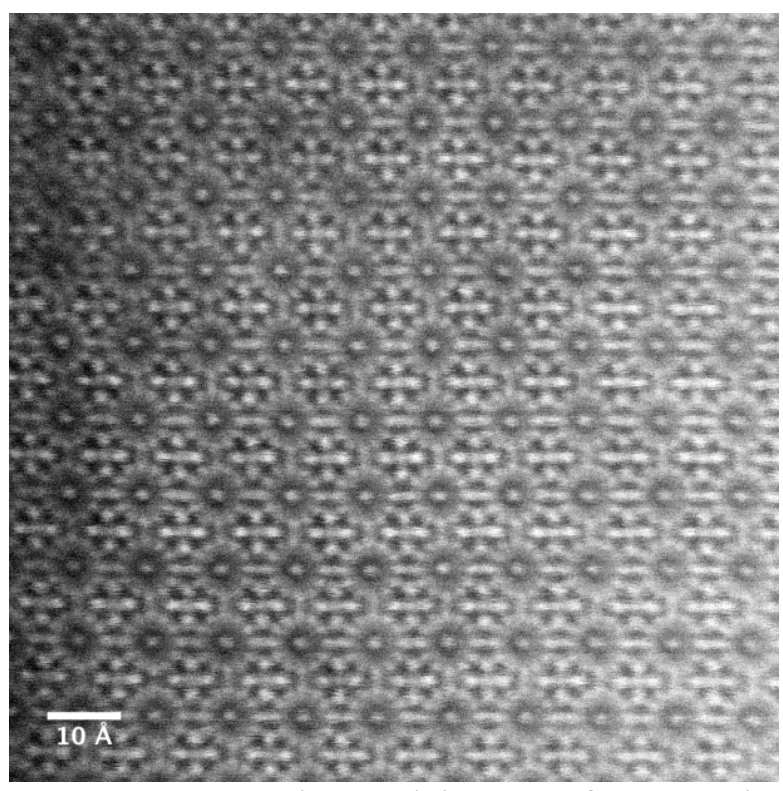

Figure 1: Experimental image of a $\mathrm{K}_{8-\mathrm{X}} \mathrm{Si}_{46}$ type I silicon clathrate along the [100] axis acquired on the $\mathrm{NCEM} \mathrm{C}_{\mathrm{s}}$-corrected VGHB501. Thickness variations, noise and small depth of focus make direct quantification difficult.
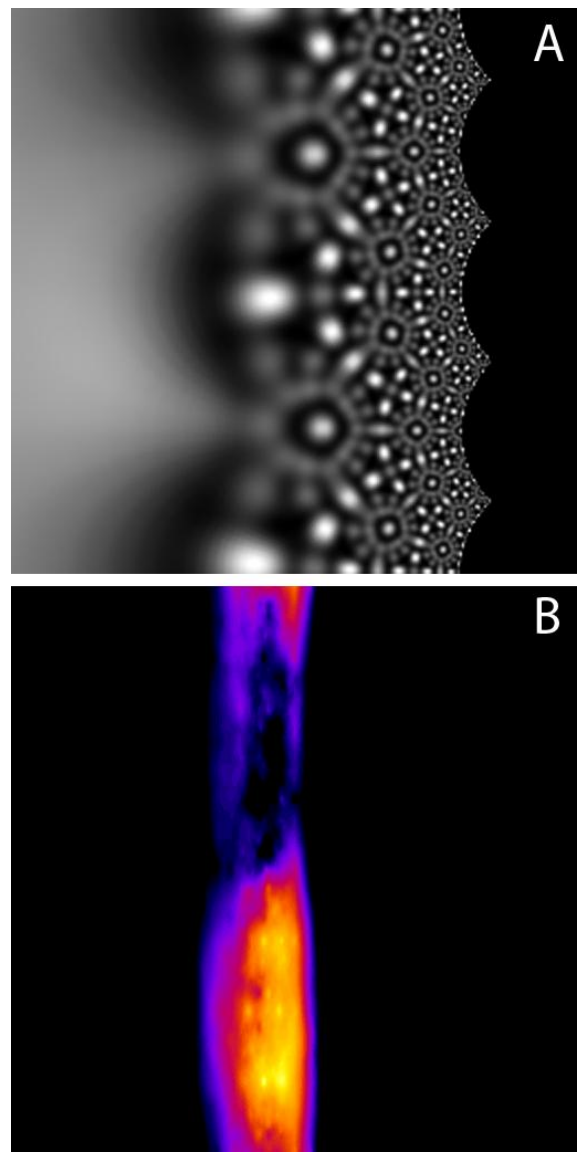
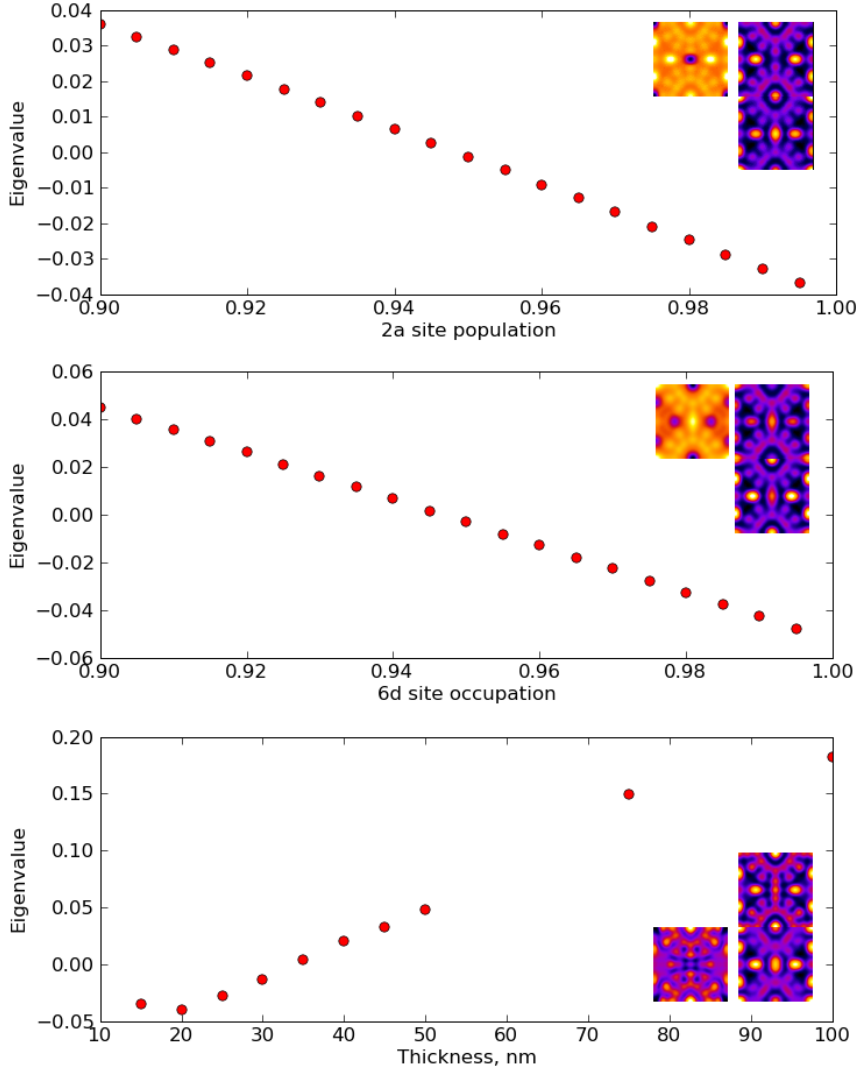

Figure 2: Eigenvalues derived from multivariate analysis of multislice simulated HAADF-STEM images along the [100] orientation of a type I silicon clathrate. Composition variations are easily identified, and thickness effects are shown to be linear in the thickness range used for highresolution imaging. Insets are the derived eigenimage (left) and importance images. Eigenimages represent approximately $1 \mathrm{~nm}^{2}$.

Figure 3 (left): A) Log-polar transformed image of the silicon clathrate. Changes to scale and rotation appear as phase shifts in this image. B) Cross correlation of a transformed reference with a transformed experimental image. The point of maximum correlation indicates the adjustment necessary to bring the images into registration. 